FERMILAB-CONF-04-393-E

CDF/PUB/TOP/PUBLIC/7287

Version 1.0

October 13, 2004

ichep2004-proc'11-0107: submitted to World Scientific on October 13, 2004 


\title{
TOP PRODUCTION CROSS SECTION FROM CDF
}

\author{
ANYES TAFFARD \\ FOR THE CDF COLLABORATION \\ University of Illinois at Urbana-Champaign, 1110 W. Green Street, Urbana, IL 61801, USA \\ E-mail: anyes@uiuc.edu
}

Recent top physics results from the CDF at a center-of-mass energy of $1.96 \mathrm{TeV}$ are presented. Measurements of the $t \bar{t}$ production cross section in all three decay channels, using a set of complementary experimental methods, are presented as well as results of a search for single top production.

\section{Introduction}

Since the discovery of the top quark in 1995 by the $\mathrm{CDF}$ and $\mathrm{D} \emptyset$ collaborations $^{1}$, an extensive program to fully determined the properties of this fundamental particle of the Standard Model is underway. These properties are important not just to characterize the top quark, but also for the understanding of its relationship to other particles in the model. Many theoretical models, that attempt to circumvent the theoretical short comings of the Standard Model, identify the top quark as a possible window into electroweak symmetry breaking and predict the existence of new particles that would affect top physics.

Run II at the Fermilab Tevatron, with proton-antiproton collisions at $\sqrt{s}=$ $1.96 \mathrm{TeV}$, offers a unique opportunity to do precision top physics. With the increase in center of mass energy with respect to Run I, the $t \bar{t}$ pair production cross section is expected to increase by 30 to $40 \%$. The study of the top quark at $\mathrm{CDF}^{2}$, also provides a testbench for tools and techniques which will be useful for physics analysis at the LHC.

\section{Top Quark Properties in the Standard Model}

Top quarks are pair-produced at the Tevatron via quark-antiquark annihilation (85\%) or via gluon fusion $(15 \%)$, with a NLO cross section ${ }^{3}$ of $6.7_{-0.9}^{+0.7} \mathrm{pb}^{3}$ for $M_{\text {top }}=$
$175 \mathrm{GeV} / \mathrm{c}^{2}$; single top production is suppressed by a factor of two relative to $t \bar{t}$ production. The large mass of the top quark has a significant impact on its phenomenology. Unitary constraints in the CKM matrix require large values for $V_{t b}$, thus the top quark decays before hadronization to a $\mathrm{W}$ boson and a b quark.

The experimental signature of a $t \bar{t}$ event is $2 \mathrm{~b}$ jets along with the decay products from the two $\mathrm{W}$ bosons. Each $\mathrm{W}$ decays either to a charged lepton and a neutrino that remains undetected, or to a quark-antiquark pair that appears in the detector as jets. The decay mode of the W's are used to categorize $t \bar{t}$ events into dilepton $\left(l^{+} \nu b l^{-} \bar{\nu} \bar{b}, \sim 11 \%\right.$ of all $t \bar{t}$ events), lepton + jets $\left(l \nu b q \overline{q^{\prime}} \bar{b}, \sim 44 \%\right)$ and all hadronic $\left(q \bar{q}^{\prime} b q \bar{q} \bar{q}^{\prime} \bar{b}, \sim 44 \%\right)$ channels.

\section{Top Quark Pair Production Cross Section}

At CDF, we measure the $t \bar{t}$ production cross section in all three categories using either counting experiments or by fitting the data to kinematics distributions that can discriminate background from signal.

\subsection{Dilepton Channel}

The dilepton channel is characterized by two leptons with high transverse momentum $\left(P_{T}\right)$ and missing energy $\left(E_{T}\right)^{4}$ from the undetected neutrinos and two jets from the $b$ quarks. This final state has the highest signal 
to noise ratio but suffers from limited statistics. We currently use three different methods to measure the cross section in the dilepton channel.

The first analysis is a counting experiment similar to what was used in Run I, in which two well identified isolated, high- $P_{T}$ electrons or muons are selected. The second analysis does not require lepton identification on the second lepton but only asks for an isolated high- $P_{T}$ track. This increases the overall acceptance as well as acceptance from $\tau$ via their one-prong hadronic decay. Both methods measure the cross section using $\sim 200 \mathrm{pb}^{-1}$ of data by counting the background subtracted signal and correcting for detector acceptance and reconstruction efficiencies $^{5}$. Both measurements are consistent with one another and with the Standard Model predictions and the combined cross section obtained is:

$$
\sigma_{t \bar{t}}=7.0_{-2.1}^{+2.4}(\text { stat. })_{-1.2}^{+1.7}(\text { sys. }) \mathrm{pb}
$$

In the third method ${ }^{6}$, we take advantage of the different kinematic behavior of $t \bar{t}$ events with respect to other Standard Model processes in the two-dimensional $E_{T}$ and jet multiplicity space. A simultaneous fit of the data to the expected kinematic shapes from signal and background templates is performed using $193 \mathrm{pb}^{-1}$ of data. The fit treats $t \bar{t}, \mathrm{WW}$ and $Z \rightarrow$ $\tau \tau$ as three distinct signal processes and $\mathrm{WZ}, \mathrm{ZZ}, Z \rightarrow e e$ and $Z \rightarrow \mu \mu$ as one combined background shape. From the result of this fit, we extract a $t \bar{t}$ production cross section of $8.6_{-2.1}^{+2.5}$ (stat.) $)_{-1.1}^{+1.1}$ (sys.) pb and a WW production cross section of $12.6_{-3.0}^{+3.2}$ (stat. $)_{-1.2}^{+1.2}$ (sys.) pb.

\subsection{Lepton+Jets Channel}

Analyses in the lepton+jets channel select events in which one $\mathrm{W}$ decays leptonically to electron or muon. The sample size is larger than the dilepton sample, but there is a significant background contamination from
QCD $W+$ jets. The purity of the sample can be improved by the identification of at least one b-jet. We currently have developed two different b-tagging algorithms. The first one makes used of the long lifetime of the $b$ quarks to reconstruct a displaced secondary vertex (SVXtagger). The second one (SLTtagger) identifies the soft muon from semileptonic b-decay. The signal to noise ratio can be further improved by requiring the scalar sum of the energy in the event, $H_{T}$, to be greater than $200 \mathrm{GeV}$.

\section{Counting experiments}

By selecting lepton + jets events with three or more jets, $H_{T}>200 \mathrm{GeV}$ and at least of the jets tagged as a b-jet by the $S V X$ tagger $^{7}$, the measured cross section from $162 \mathrm{pb}^{-1}$ of data is:

$$
\sigma_{t \bar{t}}=5.6_{-1.0}^{+1.2}(\text { stat. })_{-0.7}^{+1.0}(\text { sys. }) \mathrm{pb}
$$

If we use the SLTtagger 8 instead of the SVXtagger, the result obtained using $193 \mathrm{pb}^{-1}$ of data yields to a $t \bar{t}$ production cross section measurement of:

$$
\left.\sigma_{t \bar{t}}=4.2_{-1.9}^{+2.9} \text { (stat. }\right)_{-1.4}^{+1.4} \text { (sys.) pb }
$$

We also measure the $t \bar{t}$ cross section using events where two jets have been tagged with the SVXtagger. Since this sample provides "golden" events for the measurement of the top mass, due to the reduced combinatorics from the jet-parton match, we have developed a "looser" SVXtagger? which has a higher b-tagging efficiency at the expense of a higher rate of mistags from light quark jets. The measured cross section using the "standard" and "looser" SVXtagger are given in equations 4 and 5 respectively.

$$
\begin{aligned}
\sigma_{t \bar{t}} & \left.=5.4_{-1.9}^{+2.4} \text { (stat. }\right)_{-0.9}^{+1.1} \text { (sys.) pb } \\
\sigma_{t \bar{t}} & \left.=8.2_{-2.1}^{+2.4} \text { (stat. }\right)_{-1.0}^{+1.8} \text { (sys.) pb }
\end{aligned}
$$

\section{Kinematic fits}

Instead of counting signal and background events, one can extract the fraction of $t \bar{t}$ 
events in the lepton+jets sample by fitting one or more kinematic variables in the data to the expected shapes from signal and backgrounds. We present three different methods ${ }^{10} 11$ using this technique.

The first method uses the discriminating power of $H_{T}$ while the second method uses a neural network trained on seven kinematic variables to extract the $t \bar{t}$ fraction in the data. Note that neither of the methods require b-tagging. The $t \bar{t}$ cross section using $193 \mathrm{pb}^{-1}$ of data are measured to be $4.7_{-1.6}^{+1.6}$ (stat. $)_{-1.8}^{+1.8}$ (sys.) $\mathrm{pb}$ and $6.7_{-1.1}^{+1.1}$ (stat. $)_{-1.6}^{+1.6}$ (sys.) pb respectively.

The third method requires at least one jet tagged with SVXtagger. The kinematic variable used for the template is the $E_{T}$ of the highest $E_{T}$ jet in the event. This method, using $162 \mathrm{pb}^{-1}$ of data, yields to a cross section of:

$$
\sigma_{t \bar{t}}=6.0_{-1.8}^{+1.5}(\text { stat. })_{-0.8}^{+0.8}(\text { sys. }) \mathrm{pb}
$$

\subsection{All Hadronic Channel}

The all hadronic channel provides a very large event sample but is very challenging due to the overwhelming multi-jet QCD background. The poor signal to noise ratio of 1:2500 is improved to $1: 4$ by topological cuts, a cut on the total energy in the event and at least one jets tagged with SVXtagger. The cross section is measured by counting signal and expected background in events with six to eight jets ${ }^{12}$. The extracted cross section from $165 \mathrm{pb}^{-1}$ of data is:

$$
\sigma_{t \bar{t}}=7.8_{-2.5}^{+2.5}(\text { stat. })_{-2.3}^{+4.7}(\text { sys. }) \mathrm{pb}
$$

\subsection{Cross Section Summary}

Results from the various top quark pair production cross section are summarized in figure 1. All results are found to be consistent with each other and with the Standard Model prediction $^{3}$.

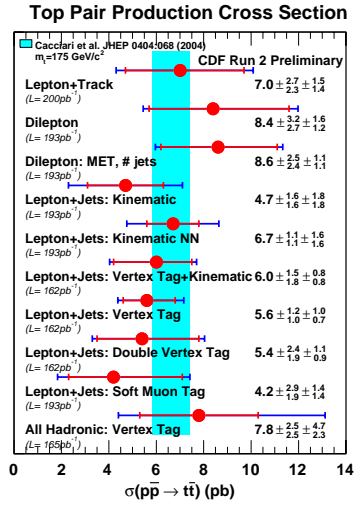

Figure 1. Summary of the measured $t \bar{t}$ cross section from CDF. The band indicated the theory expectation.

\section{Single Top}

Within the Standard Model, top quarks are also expected to be produced singly by the electroweak interaction involving a Wtb vertex. At the Tevatron, the two relevant modes are the t-channel through $\mathrm{Wg}$ fusion and the s-channel via a virtual $W$. The measurement of the single top production is particularly interesting since it would provide a direct determination of $\left|V_{t b}\right|$ but also offer sensitivity to new physics, particularly in the s-channel, to the production of new charged gauge bosons, and in the t-channel to anomalous couplings and flavor changing neutral currents. The extraction of a single top signal is challenging due to the $\mathrm{W}+$ jets background.

The event selection consists of isolating events with a $\mathrm{W}$ and two jets, one of which is tagged as a b-jet with the SVXtagger. We perform two separate searches in $162 \mathrm{pb}^{-1}$ of data $^{13}$, one for the combined s- and t-channel to optimize sensitivity to a single top signal, and a separate search to measure the rate of the two single top processes individually thus increasing sensitivity to new physics. For the combined search a likelihood fit to the $H_{T}$ distribution is used. For the separate search, the distribution of $Q_{l} \times \eta_{q}$, where $Q_{l}$ is the 
charge of the lepton from the $\mathrm{W}$ decay and $\eta_{q}$ is the pseudorapidity of the untagged jet, is used since it exhibits a distinct asymmetry for the t-channel. We find no evidence of electroweak single top quark production and set upper limits of $10.1 \mathrm{pb}$ at $95 \%$ C.L. for the t-channel cross section, $13.6 \mathrm{pb}$ for the schannel and $17.8 \mathrm{pb}$ for the combined search.

\section{Summary and Conclusions}

In this article preliminary measurements of the top pair production cross section and limits on the single top production cross sections were presented using a data set twice as large as the Run I data set. All the measurements are found to be in agreement with the Standard Model expectation but are still statistically limited. As the amount of Run II data collected increases and the systematic uncertainties determined from data decrease, $\mathrm{CDF}$ will will be able to achieve even more precise measurements of the top quark properties and probe for physics beyond the Standard Model.

\section{Acknowledgments}

I would like to thank the organizers for this very enjoyable conference and their hospitality. I also would like to thanks my colleagues in the CDF top physics group whose work went into the results presented here.

\section{References}

1. F.Abe et al. (CDF Collaboration), Phys Rev. D50 2966 (1994); Phys Rev. Lett 742626 (1995).

S.Abachi et al. (D0 Collaboration), Phys Rev. Lett 742632 (1995).

2. F. Abe et al. Nucl. Instrum. Methods Phys. Res. A 271, 387 (1988); D. Amidei et al., Nucl. Instrum. Methods Phys. Res. A 350, 73 (1994); F. Abe et al. Phys Rev. D52 4784 (1995);
P.Azzi et al. Nucl. Instrum. Methods Phys. Res. A 360, 137 (1995); The CDFII Detector Technical Design Report, Fermilab-Pub-96/390-E.

3. N. Kidonakis and R. Vogt, Phys Rev. D68 114014 (2003).

M. Cacciari, S. Frixione, M. L. Mangano, P. Nason and G. Ridolfi, JHEP 0404, 068 (2004).

4. CDF uses a cylindrical coordinate system in which $\theta$ is the polar angle with respect to the proton beam direction, $\phi$ is the azimutal angle about the beam axis, and pseudorapidity is defined as $\eta \equiv-\ln \tan (\theta / 2)$. The transverse energy and transverse momentum are defined as $E_{T}=E \sin \theta$ and $P_{T}=P \sin \theta$, respectively, where $E$ is the energy measured in the calorimeter and $P$ is the momentum measured by the tracking system. Missing transverse energy, $\not_{T}$, is defined as the vector sum of the $E_{T}$ over all the calorimeter towers.

5. CDF Collaboration, D. Acosta et al. Phys Rev. Lett 93142001 (2004).

6. www-cdf.fnal.gov/physics/new/top/ confNotes/cdf7192_aida_public.ps.

7. CDF Collaboration, D. Acosta et al. Measurement of the $t \bar{t}$ production cross section in the $p \bar{p}$ colisions at $\sqrt{s}=$ $1.96 \mathrm{TeV}$ using lepton+jets events with secondary vertex b-tagging. To be submitted to Phys Rev. D.

8. www-cdf.fnal.gov/physics/new/top/ confNotes/cdf7174_SLT_200pb1_public.pdf

9. www-cdf.fnal.gov/physics/new/top/ confNotes/cdf7186_looseDoubleTagXSPub.ps

10. www-cdf.fnal.gov/physics/new/top/ confNotes/cdf7154_ttxs_kin_pub.ps

11. CDF Collaboration, D. Acosta et al. Measurement of the $t \bar{t}$ production cross section in the $p \bar{p}$ colisions at $\sqrt{s}=$ $1.96 \mathrm{TeV}$ using kinematic fitting of $b$ tagged lepton+jets events. To be submitted to Phys Rev. D. 
12. www-cdf.fnal.gov/physics/new/top/ confNotes/cdf7075_conf_note_allhad.ps

13. CDF Collaboration, D. Acosta et al. Search for electroweak single top quark production in ppbar collisions at $\sqrt{s}=$ $1.96 \mathrm{TeV}$. To be submitted to Phys Rev. Lett. 